\title{
Predicting the outcomes and costs for a cohort of 426 patients with Chronic Obstructive Pulmonary Disease (COPD) in Bulgaria through a Markov model
}

\author{
Konstantin Tachkov ${ }^{1}$, Konstantin Mitov ${ }^{1}$, Alexandra Savova ${ }^{1}$ \\ 1 Faculty of Pharmacy, Medical University of Sofia, Department of Organization and Economy of Pharmacy, Sofia, Bulgaria \\ Corresponding author: Konstantin Tachkov (Faculty of Pharmacy, Medical University of Sofia, Department of Organization and Economy of \\ Pharmacy 1000, 2 Dunav Str., Sofia, Bulgaria. E-mail: tachkov@outlook.com; Tel: +359 8784498 55)
}

Received 4 April 2019 • Accepted 22 April 2019 • Published 9 July 2019

Citation: Tachkov K, Mitov K, Savova A (2019) Predicting the outcomes and costs for a cohort of 426 patients with Chronic Obstructive Pulmonary Disease (COPD) in Bulgaria through a Markov model. Pharmacia 66(2): 53-57. https://doi.org/10.3897/pharmacia.66.e35162

\begin{abstract}
The aim was to estimate the economic burden, as well as rate of progression of COPD for a cohort of 426 patients for a 10-year period. A total of 426 patients from 19 regions with Chronic Obstructive Pulmonary Disease were enrolled in a representative, ambispective, national study for Bulgaria. Patients were recorded on disease stage, occupation, smoking habits and medication. Cost of treatment was calculated and a 10-year one-way Markov model was used by employing transition probabilities and quality of life data from available literature. Costs and outcomes were recorded and a cost-effectiveness acceptability curve (CEAC) was established.

Out of all patients included in the study 288 were non-smokers with a mortality percentage after 10 years of $42.7 \%$. Smokers showed faster transition rate with 139 of them transitioning to more severe states and mortality was $54.6 \%$. The incremental cost-effectiveness ratio (ICER) was 863.75 BGN. Patients who smoked had a lower quality of life - cumulatively for the 10-year period QALY = 623.51 for smokers vs. 1557.51 for non-smokers, but also lower costs for treatment (538 007.52 BGN vs. 1344757.95 BGN) accounted by the high transition rate and mortality amongst that group. Relatively few non-smokers transitioned from a less severe to a more severe state (6\%), while most of the very severe stage patients experienced a lethal outcome within 10 years (81\%).

Despite the higher costs associated with the disease, the Quality of Life and lower transition probability would enable patients to live a normal life. The ICER was well below the WHO threshold.
\end{abstract}

\section{Keywords}

COPD, Markov Model, Pharmacotherapy, Cost-effectiveness

\section{Introduction}

Chronic Obstructive Pulmonary Disease (COPD) is rapidly becoming a hugely significant disease, with esti- mates pointing towards it becoming the second most common diagnosis by 2030 (Murray et al. 1996). Currently, 4 million people die annually and this number is also expected to increase, making it the $4^{\text {th }}$ leading cause 
of death (Bousqet and Khaltaev 2007). Furthermore, it is defined by the Global Initiative for Chronic Obstructive Pulmonary Disease (GOLD) as a "common, preventable and treatable disease that is characterized by persistent respiratory symptoms and airflow limitation due to exposure to noxious particles and gases", which makes timely diagnosis and treatment a priority.

The challenge in tackling the disease is two-fold. On the one hand, the ageing population of European Countries, in combination with new medicines and medical devices which prolong life, has increased the frequency of people developing chronic diseases (Yach et al. 2004). On the other hand, it is a disease more colloquially known as "smokers' disease" and patient decisions influence to a great extent its progression, with ex-smokers experiencing significant lung-function improvement over the years (Scanlon et al. 2000). Thus, effective strategies in managing and preventing the disease require timely diagnosis, appropriate treatment and extensive patient education.

Bulgaria suffers in all 3 aforementioned aspects. A total of $39 \%$ of the adult population over 40 year of age are smokers (Ministry of Health 2015), limited healthcare resources allow for full reimbursement for some COPD medicines and partial for other, which influences prescribing practices (Chandra et al. 2012) and patients, more often than not, wait until symptoms manifest before they visit a General Practitioner (GP), who subsequently refers them to a specialist physician, resulting in late diagnoses.

The field of COPD in Bulgaria also has not been studied recently in depth (Pavlov et al. 2012, Andreevska et al. 2010a, b, Kyuchukov et al. 2016). There are no patient registers and, to our knowledge, no substantial follow-up study has been done on a large cohort of patients. Furthermore, there are no long-term prospective studies investigating the progression for patients in the country, as well as their habits. This paper will attempt to simulate the natural progression of disease in our cohort and address the aforementioned inadequacies in knowledge, as well as provide some much-needed information on patient costs and outcomes. This model will also serve as the basis for future research into the effect of COPD on the wider Bulgarian population.

The aim was to estimate the economic burden, as well as the rate of progression of COPD for a cohort of 426 patients for a 10 -year period.

\section{Materials and Methods}

\section{Design of data collection}

In 2015, 426 patients were recruited from Bulgaria with a diagnosis of COPD, with every $5^{\text {th }}$ patient, who entered a doctor's practice, asked to participate. After signing consent forms, patients were followed throughout the year and information on pharmacotherapy, smoking status, age, sex, FEV1/FEC, occupation and disease stage was collected.

\section{Cost and utility data}

The Global Initiative for COPD (GOLD) has classified the disease into 4 stages corresponding to GOLD A, GOLD $B$, GOLD C and GOLD D. The cost of treatment for each GOLD group (A, B, C and D) (Kamusheva et al. 2017) along with utility values from other literature sources was used and applied (Chandra et al. 2012) to an already constructed one-way Markov Model, which would follow the cohort for 10 years (Tachkov et al. 2018a). The measure of the utility chosen was Quality Adjusted Life Years (QALYs), since it is an appropriate choice for chronic diseases where quality and quantity of life need to be taken into account.

\section{Structure of the model}

The constructed model, previously expanded in another publication (Tachkov 2018), was one-way, with no new incidence programmed into it and it consisted of the 4 GOLD groups (A, B, C and D) and death as an absorbing state. Three-month transition probabilities were taken from Menn et al. 2012 and Tachkov et al. 2018a and transformed into annual probabilities. Table 1 shows our cohort's initial distribution and year 0 .

In this study we explored what would happen to the enrolled patients. The model was set to run for 10 years and the Incremental Cost-Effectiveness Ratio was calculated for 2 groups of patients - smokers and non-smokers. Ex-smokers were put in the non-smoker category if they had quit more than a year ago.

The mortality percentage was calculated for both subgroups in the cohort, the value of each Life Year Saved (LYS) was obtained and an ICER was calculated.

Table 1. Smokers and non-smokers initial distribution and cost and utility data.

\begin{tabular}{lccccc}
\hline & GOLD A & GOLD B & GOLD C & GOLD D & Source \\
\hline Smokers (\%) & 7 & 49 & 23 & 60 & Tachkov 2018 \\
Non-smokers (\%) & 23 & 96 & 40 & 729 & Tachkov 2018 \\
Mean therapy cost (SD) & 438 & 591 & Tachkov et al. 2017 & 790 & $(704.44-875.56)$ \\
Quality Adjusted Life & $(349.43-523.56)$ & $(505.43-676.52)$ & $(687.44-858.56)$ & 0.66 & Chandra et al. 2012 \\
Years (QALY) & 0.85 & 0.81 & & & \\
\hline
\end{tabular}




\section{Sensitivity analysis}

A deterministic (tornado diagram) and probabilistic sensitivity analysis (Monte Carlo simulation) were carried out and a cost-effectiveness acceptability curve (CEAC) was constructed. The margin of change for values was $\pm 30 \%$ for the deterministic analysis and the Monte Carlo simulation consisted of 1000 recalculations of the ICER. The CEAC showed the price range at minimum and maximum, where ICER was $0 \%$ or $100 \%$ within the scope.

\section{Results}

The majority of our patients were non-, or ex-smokers, but already in late stages of the disease. Nonetheless, they had the most control over the disease with very few patients transitioning to a more severe state - 19 patients (6\%). Mortality was high in the GOLD D subgroup (81.3\% of patients). This accounts for the high cost and utility values accumulated by non-smokers in the model (Table 2). The accumulated costs, after 10 years projection, were 1334757.95 BGN, but the sum of QALYs was 1557.51 with a cost-effectiveness ratio between the two of 963.4 BGN additional amount of money spent for additional QALY improvement. Non-smoking patients tended to live longer and experience better quality of life, allowing for slower disease progression and accumulation of benefits. Our observed CER accounts for the longer duration of life with the accompanying costs.

Smokers, on the other hand, showed faster turn-over and high mortality. A total of $91.6 \%$ of patients in GOLD $\mathrm{D}$ had experienced a lethal outcome and 21 patients (31.5\%) from GOLD A and B had progressed (Table 3). This, along with the low number of smokers (139) ac- counts for the lower accumulated costs - 538 007.52 BGN and QALYs - 623.51 with a ratio of 862.87 between the two. The faster rate of disease progression in smokers would mean that they experience a higher rate of severe exacerbations, which are a risk factor for death in COPD and lead to poorer quality of life.

The incremental ratio between the two CER values was 863.75BGN with the incremental value for each LYS being 8067.5 BGN. (Table 4) The overall mortality was $54.6 \%$ for smokers and $42.7 \%$ for non-smokers, further indicating better disease control in the non-smoker subgroup. The cost-effectiveness of the ratio showed that non-smokers experience added benefits at a reasonable price and live longer to enjoy those benefits.

Despite these differences, the Monte-Carlo cluster revealed a homogenous dispersion of data points (Figure 1), while the CEAC showed that all 1000 values became cost-effective within the scope of 820-920 BGN (Figure 2).

In terms of the deterministic sensitivity analysis, the lowest ICER for QALY was observed when the costs for non-smokers were 30\% lower (531.5 BGN) and the highest when their costs were $30 \%$ higher (1295.69 BGN). For

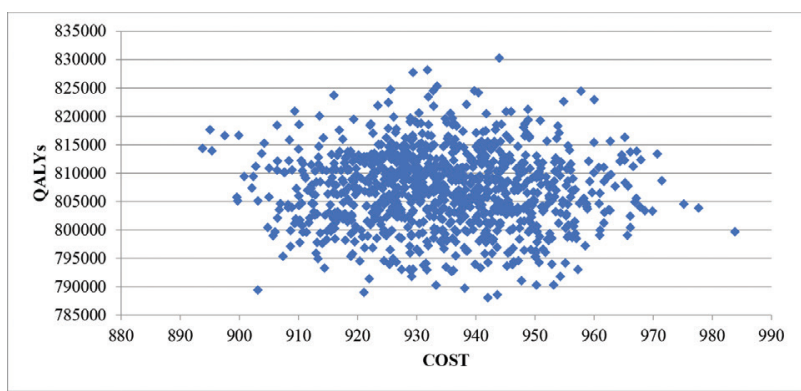

Figure 1. ICER points and dispersion cloud of Monte-Carlo simulation

Table 2. Model value results for non-smokers.

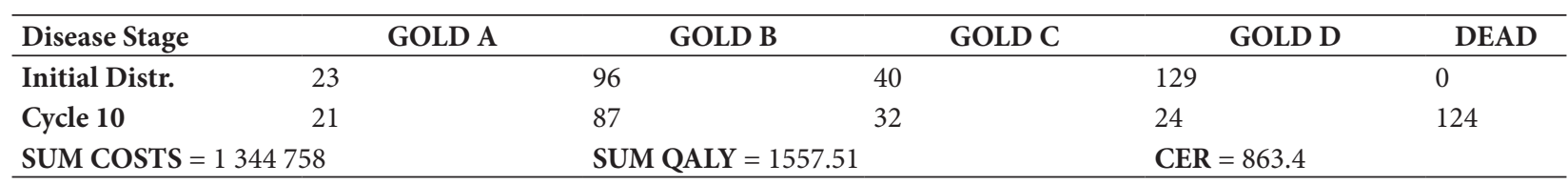

Table 3. Model value results for smokers.

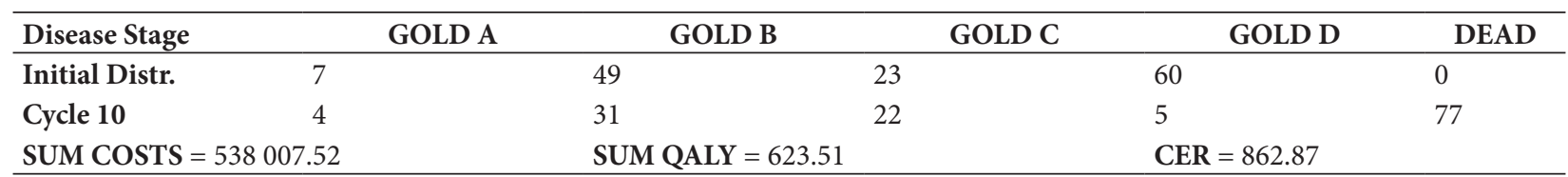

Table 4. Summarised ICER values for QALY and LYS.

\begin{tabular}{|c|c|c|c|c|}
\hline Group & SUM COSTS & SUM QALYs & CER & ICER QALY \\
\hline Non-smokers & 1344757.95 & 1557.51 & 863.402 & \\
\hline Smokers & 538007.52 & 623.51 & 862.869 & 863.76 \\
\hline \multicolumn{2}{|c|}{ GROUP SUM COSTS } & \multicolumn{2}{|c|}{ SUM LYS } & ICER LYS \\
\hline Non-smokers & 1344757.95 & 165 & 8150.048 & \\
\hline Smokers & 538007.52 & 65 & 8277.038 & 8067.50 \\
\hline
\end{tabular}




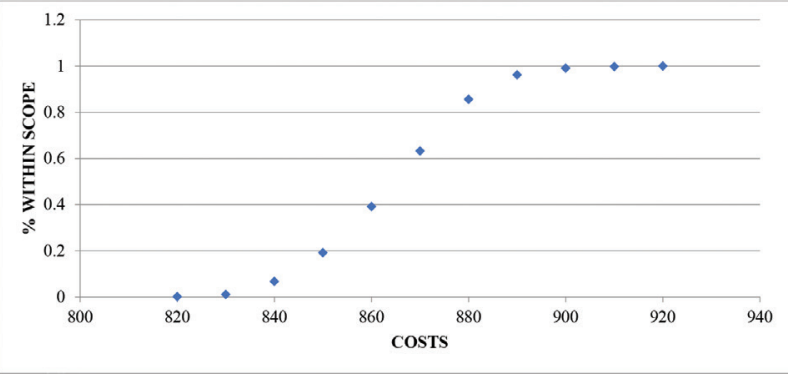

Figure 2. CEAC of all data points

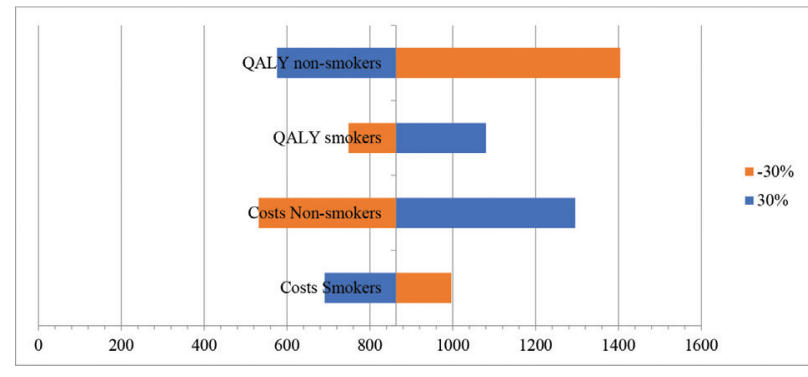

Figure 3. Tornado diagram for QALYs

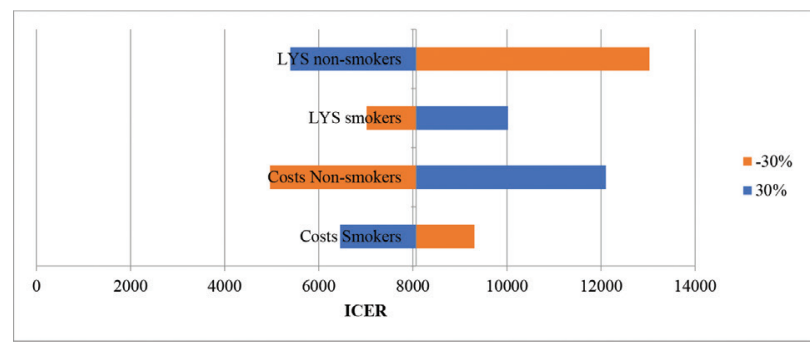

Figure 4. Tornado diagram for LYS

LYS, the minimal ICER was $4964.22 \mathrm{BGN}$ when the cost of therapy for non-smokers was 30\% lower, while maximal value was 13 028.27 BGN when LYS for non-smokers was $30 \%$ lower (Figures 3 and 4 ).

\section{Discussion}

To our knowledge, this is the first Bulgarian COPD Markov model. It tries to simulate the progression of disease in a cohort of 426 patients, based on their smoking status. It also provides information regarding which stage of disease is the most difficult to control, since large-scale observational studies are not always economically feasible, in order to obtain such information.

Patients in the studied cohort are a representative sample of the country and reveal that diagnosis in GOLD A is a rare phenomenon. Overall, only $7 \%$ of all patients in the cohort were in GOLD A. The other important aspect to be considered is that smoking status is an acute problem for the country where established data from the National Statistics Institute (NSI) show that $39 \%$ of the population over 40 smoke, and if people from all age groups are included in the statistics, the estimates show that more than $50 \%$ of the entire country are current smokers (National Statistical Institute). We have used this data, along with our model elsewhere, to predict what would be the disease prevalence and progression for the wider Bulgarian population, coupled with the costs and outcomes (Tachkov 2018). The results from our current model were interesting and present a hypothetical view of how the next 10 years would unfold for the patient, for whom we had only one year of observation. Despite the short follow-up period, our results are consistent with documented literature on how smoking affects the natural progression of COPD (Bai et al. 2017) and serve as a validation to the model. The implication from these results for Bulgaria would lead us to believe that a national smoking cessation programme should be implemented, as well as encouraging patients to seek professional help at earlier stages, since Gold A patients have the best disease control of non-smokers and tend to live longer.

Thus, it is possible to conclude that the disease will have an increasing impact on Bulgarian society in the coming years. Therefore, it was necessary to evaluate the cost-effectiveness and rate of progression for both non-smokers and smokers, in order to help decision-makers and patients make more informed decisions. The main strength of the model is that it is based on real-world data, taken from real-life pulmonologist practices.

It combines this with previous studies in the field on lung function decline and benefits of adequate disease control (Pavlov et al. 2012) and has constructed the first model of this kind for the country, allowing for predictions to be made on for the general population, as well as for different patient cohorts. The preliminary results from the model in the article were also presented as an Abstract at the ISPOR annual European Congress in Barcelona, Spain (Tachkov 2018).

A limitation of the model is the fact that it is a one-way, static model and cannot draw definite conclusions on whether or not smoking cessation is achieved in all people afflicted by the disease. Due to the model's nature, costs remained stable, thus only one aspect of the disease was reflected, not accounting for the possibility of back-transitions. The high variation of $30 \%$ in the sensitivity analysis was used to compensate for this shortcoming and it is worth pointing out, that in all cases, the ICER was well below the established threshold of 3 times GDP per capita. This would indicate that the cost-effectiveness of treatments can allow for an addition of a prevention programme.

The other limitation is the short term, real-life observation of the patients for a 2 year time period.

This work is a logical and scientific development of the already published study (Tachkov 2018). The first study is testing the hypothesis of cost-effectiveness of the COPD therapy for the general population and this study is predicting the pharmacotherapy results for a particular patient cohort. Both studies confirm that the constructed model could be used for a real life prediction of the cost-effectiveness of COPD pharmacotherapy. In addition, both validated the results that the treatment of smokers and non-smokers is cost - effective for the Bulgarian population. 


\section{Conclusion}

Despite the higher costs associated with the disease, the Quality of Life and lower transition probability would enable non-smokers to live longer lives with better disease control. The ICER was well below the WHO threshold making

\section{References}

Andreevska K, Dimitrova Z, Kofinov R, Petkova V (2010a) Evaluation of COPD treatment patterns for pulmonologists in Bulgaria. Trakia Journal of Sciences 8(2): 358-364.

Andreevska K, Petkova V, Dimitrova Zl (2010b) Medico social analysis of the prevalence of COPD. Scripta Periodica 13(2): 9-20

Bai JW, Chen XX, Liu S, Yu L, Xu JF (2017) Smoking cessation affects the natural history of COPD. International Journal of Chronic Obstructive Pulmonary Disease 12: 3323-3328. https://doi. org/10.2147/COPD.S150243

Bousqet J, Khaltaev N (2007) Global Surveillance, Prevention and Control of Chronic Respiratory Diseases, A Comprehensive Approach. World Health Organization Press, Geneva, 155 pp. https:// www.who.int/gard/publications/GARD\%20Book\%202007.pdf

Chandra K, Blackhouse G, McCurdy BR, Bornstein M, Campbell K, Costa V, Franek J, Kaulback K, Levin L, Sehatzadeh S, Sikich N (2012) Cost-effectiveness of interventions for COPD using Ontario Policy Model. Ont Health Technol Assess Ser 12(12): 1-61. https:// www.ncbi.nlm.nih.gov/pmc/articles/PMC3384363/

Kamusheva M, Dimitrova M, van Boven JF, Postma MJ, van der Molen T, Kocks JW, Mitov K, Doneva M, Petrova D, Georgiev O, Petkova V (2017) Clinical characteristics, treatment patterns, and socio-economic burden of COPD in Bulgaria. Journal of Medical Economics 20(5): 503-509. https://doi.org/10.1080/13696998.201 7.1279620

Kyuchukov N, Pavlov P, Glogovska P, Popova T, Yanev N, Krachunov I, Ivanova Z, Ivanov Y, Hristova P (2016) Hospital treatment costs of exacerbations in COPD patients with indications for LTOT. European Respiratory Journal 46: PA1053. http://doi. org/10.1183/13993003.congress-2015.PA1053

Menn P, Leidl R, Holle R (2012) A lifetime Markov Model for the economic evaluation of Chronic Obstructive Pulmonary disease. Pharmacoeconomics 30(9): 825-840. https://doi.org/10.2165/11591340000000000-00000 the cost per additional benefits cost-effective. For patients who want to achieve adequate disease control, smoking cessation is necessary, since smokers have higher mortality rates. A broader burden of disease study from a societal perspective is necessary to estimate the savings that would be achieved from a smoking-cessation programme.

Ministry of Health (2015) Yearly report on the health condition of the citizens of Republic Bulgarian and fulfillment of the health strategy. http:// ncphp.government.bg/files/doklad_zdrave_-17-10.pdf

Murray CJL, Lopez AD, World Health Organization, World Bank, Harvard School of Public Health (1996) The Global burden of disease: a comprehensive assessment of mortality and disability from diseases, injuries, and risk factors in 1990 and projected to 2020. Harvard University Press, 41 pp. http://www.who.int/iris/handle/10665/41864

Pavlov P, Ivanov Y, Glogovska P, Popova T, Borisova E, Nozharov V (2012) New epidemiological data for COPD in Pleven region. Thoracic Medicine 4(2): 44-50.

Scanlon PD, Connett JE, Waller LA, Altose MD, Bailey WC, Sonia Buist A, e Lung Health Study Research Group DP (2000) Smoking Cessation and Lung Function in Mild-to-Moderate Chronic Obstructive Pulmonary Disease. The Lung Health Study. American Journal of Respiratory and Critical Care Medicine 163: 381-390 http://doi.org/10.1164/ajrccm.161.2.9901044

Tachkov K, Kamusheva M, Pencheva V, Mitov K (2017) Evaluation of the economic and social burden of chronic obstructive pulmonary disease (COPD). Biotechnology \& Biotechnological Equipment 31(4): 855861. https://doi.org/10.1080/13102818.2017.1335616

Tachkov K, Mitov K, Savova A, Kamusheva M (2018a) A one-way, static Markov model estimating the social and economic burden of chronic obstructive pulmonary disease in Bulgaria for patients who smoke or don't. Biomedical Research 29(16): 3261-3266. https://doi. org/10.4066/biomedicalresearch.29-18-938

Tachkov T, Savova A, Mitov K (2018b) PRS15 - a 10-year follow-up of a cohort of patients with chronic obstructive pulmonary disease (COPD) Through a one-way Markov model. Value in Health 21: S406. https://doi.org/10.1016/j.jval.2018.09.2409

Yach D, Hawkes C, Gould CL, Hofman KJ (2004) The global burden of chronic diseases: overcoming impediments to prevention and control. JAMA 291(21): 2616-26225. http://doi.org/10.1001/jama.291.21.2616 